\title{
Biomolecular Analysis of Juvenile Nasopharyngeal Angiofibroma
}

\section{Muhammet Recai Mazlumoglu*}

Otorhinolaryngology Clinic, Hinis Sehit Yavuz Yurekseven State Hospital, Erzurum-25600, Turkey

\begin{abstract}
Juvenile nasopharyngeal angiofibroma (JNA), a rare type of tumor that occurs most frequently in adolescent males, is difficult to treat. The relationship between JNA and certain biomolecules has been the subject of a great deal of research conducted over a long period. This review summarizes the research on the etiopathogenesis of JNA.
\end{abstract}

Keywords: Nasopharyngeal angiofibroma; Juvenile; Biomolecule; Cancer; Genetic

\section{Introduction}

Juvenile nasopharyngeal angiofibroma (JNA) is a benign tumor of the nasopharynx, which accounts for $0.5 \%$ of all head and neck tumors. Although it can occur in all age groups, it is most common in adolescents and young adults between 14 and 25 years of age. It is also more common in males than in females [1].

Although JNA is histologically benign, it shows locally invasive behavior and is treated surgically. However, recurrence rates are high, at up to $42 \%$ [2]. The most common complication of surgery is bleeding. Therefore, studies on JNA have focused on the difficulty of surgical treatment and high relapse rate. Primary or adjuvant therapy targeting biomolecules secreted from tumor tissue in large quantities (molecular-targeted therapy) can help to reduce the rates of complications, can be used primarily in the treatment, can render otherwise inoperative tumors amenable to surgical intervention, and can be used in palliative treatment.

\section{Literature Review}

The pathogenesis of JNA is still unknown. There have been a number of studies regarding tumor formation and growth in JNA, and regarding its prognostic. These studies took into consideration the male predominance of the tumor, its vascular nature, and its common onset in adolescence. The roles of growth factors, hormone receptors, tumor suppressor genes, oncogenes, and cytokines in the etiopathogenesis of JNA have all been evaluated (Table 1) [2-42].

JNA-related molecular studies have focused on sex hormone receptors, with investigations being done of androgen receptors (AR), estrogen receptors (ER), and progesterone receptors (PR) $[6,10,14,18$ $24,39,40]$. These studies indicated that AR is frequently expressed at high levels in cases of JNA [18-22]. However, some studies indicated that ER and PR expression levels are high, while those of AR are low, in JNA $[24,39,40]$. Kumagami et al. conducted two studies on this subject $[39,40]$. In both of them, ER was over-secreted by JNA tissue.

Excessive release of growth factors is a seen frequently in the development of JNA. Therefore, vascular endothelial growth factor (VEGF), nerve growth factor (NGF), insulin-like growth factor (IGF), fibroblast growth factor (FGF), platelet-derived growth factor (PDGF), and transforming growth factor (TGF) were assessed in many studies $[2,5,6,10,12,14-16,23,25-27]$. The levels of TGF- $\beta 1[10,25,27,29]$, basic fibroblast growth factor (bFGF) $[6,15,25,26]$, NGF (2), and VEGF $[5,6,10,12,14,16,23,25]$ were found to be elevated in JNA. The most notable of which are TGF- $\beta 1$ and VEGF. Dillard et al. evaluated only TGF- $\beta 1$ in one study. They reported over-expression of TGF- $\beta 1$. Saylam et al. reported that both VEGF and TGF- $\beta 1$ were over-expressed.

The roles of tumor suppressor genes, oncogenes, and cancer protooncogenes, including p53, c-Myc, $\mathrm{H}-\mathrm{Ras}$, Ki-Ras, c-Kit, p130Cas, Friend leukemia integration-1 (FLI-1), endoglin, podoplanin, stromelysin-3 (ST3), tenascin-C (TNC), syndecan-2, proliferating cell nuclear antigen (PCNA), Bcl-2, Ki-67, S-100, c-Fos, GSTM1 gene, AURKA, MDM2, Her-2/neu, and Hif-1 [2,5-7,9-13,15-17,25,27,28,32$35]$, in the etiology of JNA have been investigated. Among these, c-Myc $[6,7,28]$, c-Kit $(2,6,7)$, FLI-1 [5], endoglin [5,11], TNC [9], PCNA [10,12], Ki-67 [16], GSTM1 [17,33], AURKA and MDM2 (34) were shown to be associated with JNA. Among these, GSTM1-null genotype gene seems important. Expression of GSTM1 mRNA (which encodes glutathione S-transferase M1, a protein involved in detoxification) should be normally in all human beings. When this is not expressed there is an increased risk of developing some malignancies [17,42]. Two studies performed on GSTM1-null genotype [17,33]. In both studies, the GSTM1-null genotype was associated with the formation of JNA.

\section{Discussion}

Some authors have suggested that cellular structural proteins are involved in the etiopathogenesis of JNA. For example, E-cadherin, $\mathrm{N}$-cadherin, $\alpha$-catenin, $\beta$-catenin, $\gamma$-catenin, bone morphogenic protein 4 (BMP4), MNF116, CAM5.2, desmin, vimentin, calponin [2,13,14,30,31,41] have all been studied, and both $\beta$-catenin $[2,14,30,31,41]$ and vimentin [13] were shown to be associated with JNA.

In addition, the roles of Toll-like receptors (TLR 3, TLR 7, TLR 9), cell-surface antigens (CD31, CD34, CD68, CD99), matrix metalloproteinases (MMP-1, MMP-2, MMP-9, MMP-14), and certain cytokines (IL-6, IL-17) were investigated [6,8,12,13,16,36-38]. Of these, TLR 3 [8], CD34 [12], MMP 9 [36,37], IL-6, and IL-17 [6,38] were shown to be associated with JNA.

There have also been investigations regarding the associations between biomolecules and the prognosis of JNA, with ER, MMP-9, and IL-17 all being shown to be associated with poor prognosis $[23,37,38]$.

Carlos et al. postulated that human herpes virus-8 (HHV-8) and Epstein-Barr virus (EBV) may play roles in the etiology of JNA, but their results indicated no such associations of these viruses with the pathogenesis of JNA [4].

${ }^{*}$ Corresponding author: Dr. Mazlumoglu MR, Otorhinolaryngology Clinic, Hinis Sehit Yavuz Yurekseven State Hospital, Erzurum-25600, Turkey, Tel: +90 542435 5835; E-mail: dr.mazlumoglu@gmail.com

Received November 01, 2017; Accepted November 16, 2017; Published November 20, 2017

Citation: Mazlumoglu MR (2017)Biomolecular Analysis of Juvenile Nasopharyngeal Angiofibroma. J Mol Genet Med 11: 304 doi:10.4172/1747-0862.1000304

Copyright: (c) 2017 Mazlumoglu MR. This is an open-access article distributed under the terms of the Creative Commons Attribution License, which permits unrestricted use, distribution, and reproduction in any medium, provided the original author and source are credited 
Citation: Mazlumoglu MR (2017) Biomolecular Analysis of Juvenile Nasopharyngeal Angiofibroma. J Mol Genet Med 11: 304 doi:10.4172/17470862.1000304

Page 2 of 3

\begin{tabular}{|c|c|c|}
\hline Study & Marker & $\begin{array}{l}\text { Associated with JNA (Over-expressed or up-regulated, gene mutation, } \\
\text { gene nullity) }\end{array}$ \\
\hline Zhang et al. [2] & $\beta$--catenin, c-Kit (CD117), p130Cas, TGF-B3, BMP 4, NGF, IGF-1R & $\beta$--catenin (nuclear), c-Kit (CD117) (cytoplasmic), NGF (cytoplasmic) \\
\hline Carlos et al. [4] & HHV-8, EBV & - \\
\hline Nonogaki et al. [5] & $\begin{array}{l}\text { FLI-1,endoglin(CD105), podoplanin, VEGFR3, VEGFC, GLUT-1, } \\
\text { ST3, SPARC }\end{array}$ & FLI-1, endoglin (CD105), VEGFC \\
\hline Pandey et al. [6] & $\begin{array}{c}\text { c-Kit (CD117), c-Myc, VEGFA, bFGF, PDGF-A, H-Ras, AR, IL-6, } \\
\text { p53 }\end{array}$ & c-Kit (CD117), c-Myc, VEGFA, bFGF, PDGFA, H-Ras, IL-6 \\
\hline Renkonen et al. [7] & c-Kit (CD117), c-Myc, BMI-1 & c-Kit (CD117), c-Myc, BMI-1 \\
\hline Renkonen et al. [8] & TLR 3, TLR 7, TLR 9 & TLR 3 \\
\hline Renkonen et al. [9] & GLUT-1, TNC, syndecan-2 & GLUT-1, TNC \\
\hline Saylam et al. [10] & ER, PR, PCNA, VEGF, TGF-B & PCNA, VEGF, TGF-B \\
\hline Wang et al. [11] & Endoglin (CD105) & Endoglin (CD105) \\
\hline Zhang et al. [12] & VEGFR-1, VEGFR-2, PCNA, CD34 & VEGFR-1, VEGFR-2, PCNA, CD34 \\
\hline Pauli et al. [13] & $\begin{array}{l}\text { MNF116, CAM5.2, S-100, CD31, CD34, CD99, CD68, vimentin, } \\
\text { EMA, SMA,desmin, calponin, Bcl-2, c-Kit (CD117) }\end{array}$ & Vimentin \\
\hline Ponti et al. [14] & $\beta$--catenin, E-cadherin, AR, VEGFR-2 & $\beta$--catenin, VEGFR-2 \\
\hline Sun et al. [15] & PCNA, VEGF, bFGF & bFGF \\
\hline Brieger et al. [16] & VEGF, CD31, Ki67 & VEGF, Ki67 \\
\hline Gautham et al. [17] & GSTM1-null genotype & GSTM1-null genotype \\
\hline Brentani et al. [18] & $E R, P R, A R, G R$ & $\mathrm{PR}, \mathrm{AR}$ \\
\hline Lee et al. [19] & ER, PR, AR & AR \\
\hline Hwang et al. [20] & $E R, P R, A R$ & AR \\
\hline Farag et al. [21] & AR & AR \\
\hline Gatalica [22] & ER, PR, AR & AR \\
\hline Liu et al. [23] & AR, ER- $\alpha, E R-\beta, P R, V E G F$ & AR, ER- $\alpha, E R-\beta$, VEGF \\
\hline Montag et al. [24] & PR, ER- $\alpha$, ER-ss, AR & ER-ss \\
\hline Schuon et al. [25] & TGF- $\beta 1$, bFGF, VEGFR-1, VEGFR-2, Hif-1alpha & TGF- $\beta 1$, bFGF, VEGFR-2, \\
\hline Schiff et al. [26] & bFGF & bFGF \\
\hline Nagai et al. [27] & IGF-II, bFGF, VEGF, TGF- $\beta 1$, PDGF-A, PDGF-B, c-myc, c-fos, p53 & IGF-II, TGF- $\beta 1$, \\
\hline Shick et al. [28] & c-Myc & c-Myc \\
\hline Dillard et al. [29] & TGF-B1 & TGF-B1 \\
\hline Rippel et al. [30] & E-cadherin, $\mathrm{N}$-cadherin, $\alpha$-catenin, $\beta$-catenin, $\gamma$-catenin & $B$-catenin \\
\hline Abraham et al. [31] & $B$-catenin & B-catenin \\
\hline Shick et al. [32] & p53, Her-2/neu & - \\
\hline Maniglia et al. [33] & GSTM1-null genotype & GSTM1-null genotype \\
\hline Shick et al. [34] & AURKA gene, MDM2 gene & AURKA gene, MDM2 gene \\
\hline Coutinho et al. [35] & Ki-ras, H-ras & - \\
\hline Duerr et al. [36] & MMP-1, MMP-2, MMP-9, MMP-13, MMP-14, TIMP-1, TIMP-2 & MMP-1, MMP-2, MMP-9, \\
\hline Sun et al. [37] & MMP-9 & MMP-9 \\
\hline Sun et al. [38] & IL-17 & IL-17 \\
\hline Kumagami [39] & $E R, A R$ & ER \\
\hline Kumagami [40] & ER, PR, AR & ER, PR \\
\hline Valanzano et al. [41] & $\beta$--catenin & $\beta$-catenin \\
\hline \multicolumn{3}{|c|}{$\begin{array}{l}\text { TGF-ß1: Transforming Growth Factor Beta1, BMP4: Bone Morphogenic Protein 4, NGF: Nerve Growth Factor, IGF-1R: Insuline Like Growth Factor Receptor, HHV- } \\
\text { 8: Human Herpes Virus-8, EBV: Epstein-Barr Virus, FLI-1: Friend Leukemia Integration-1, VEGFR: Vascular Endothelial Growth Factor Receptor, VEGFC: Vascular } \\
\text { Endothelial Growth Factor Cognate Ligand, ST3: Stromelysin 3, GLUT: Glucose Transporter, SPARC: Secreted Protein Acid Rich In Cysteine, C-Myc: Avian Myelomatosis } \\
\text { Viral Oncogene Homolog, Bfgf: Basic Fibroblast Growth Factor, PDGFA: Platelet-Derived Growth Factor, H-Ras: Harvey Rat Sarcoma Viral Oncogene Homolog, AR: } \\
\text { Androgen Receptor, ER: Estrogen Receptor, PR: Progesterone Receptor, GR: Glucocorticoid Receptor, IL-6: Interleukin 6, BMI-1: Polycomb Complex Protein, TLR: } \\
\text { Toll-Like Receptor, TNC: Tenascin-C, PCNA: Proliferating Cell Nuclear Antigen, Flt-1: Fms-Like Tyrosine Kinase-1, Flk-1:Fetal Liver Kinase-1, GSTM1: Glutathione S } \\
\text { Transferase M1, TGF-B1: Transforming Growth Factor Beta 1, Hif-1alpha: Hypoxia Inducible Factor 1alpha, MMPS: Matrix Metalloproteinases. }\end{array}$} \\
\hline
\end{tabular}

Table 1: Biomolecular studies of juvenile nasopharyngeal angiofibroma.

\section{Conclusion}

A number of studies have indicated associations between certain biomolecules and JNA. However, molecular targeting has not yet been applied during clinical treatment. Nevertheless, these studies are promising in terms of the future development of possible alternative methods to surgical treatment. Also, the studies about JNA were summarized in this review. These studies were presented in the form of a table. This presentation can help authors who plan to study on JNA.

\section{References}

1. Makhasana JAS, Kulkarni MA, Vaze S, Shroff AS (2016) Juvenile nasopharyngeal angiofibroma. J Oral Maxillofac Pathol 20: 330

2. Zhang PJ, Weber R, Liang H, Pasha TL, Livolsi VA (2003) Growth factors and receptors in juvenile nasopharyngeal angiofibroma and nasal polyps. Arch Pathol Lab Med 127: 1480-1484.

3. Mishra A, Pandey A, Mishra SC (2017) Variable expression of molecular markers in juvenile nasopharyngeal angiofibroma. J Laryngol Otol 131(9): 752-759.

4. Carlos R, Thompson LDR, Netto AC, Pimenta LGGS, Correia-Silva JF, et al 
(2008) Epstein-Barr virus and human herpes virus-8 are not associated with juvenile nasopharyngeal angiofibroma. Head and Neck Pathol 2: 145-149.

5. Nonogaki S, Campos HG, Butugan O, Soares FA, Mangone FR, et al. (2010) Markers of vascular differentiation, proliferation and tissue remodelling in juvenile nasopharyngeal angiofibromas. Exp Ther Med 1: 921-926.

6. Pandey P, Mishra A, Tripathi AM, Verma V, Trivedi R, et al. (2016) Current molecular profile of juvenile nasopharyngeal angiofibroma: first comprehensive study from India. Laryngoscope 3: 100-116.

7. Renkonen S, Häyry V, Heikkilä P, Leivo I, Haglund C, et al. (2011) Stem cell-related proteins C-KIT, C-MYC and BMI-1 in juvenile nasopharyngeal angiofibroma - do they have a role? Virchows Arch 458: 189-195.

8. Renkonen S, Cardell LO, Mattila P, Lundberg M, Haglund C, et al. (2015) Tolllike receptors 3,7 , and 9 in juvenile nasopharyngeal angiofibroma. APMIS 123 439-444.

9. Renkonen S, Heikkilä P, Haglund C, Mäkitie AA, Hagström J (2013) Tenascin-C. GLUT-1, and syndecan-2 expression in juvenile nasopharyngeal angiofibroma: correlations to vessel density and tumor stage. Head Neck 35: 1036-1042.

10. Saylam G, Yucel OT, Sungur A, Onerci M (2006) Proliferation, angiogenesis and hormonal markers in juvenile nasopharyngeal angiofibroma. Int $\mathrm{J}$ Pediatr Otorhinolaryngol 70: 227-234.

11. Wang JJ, Sun XC, Hu L, Liu ZF, Yu HP, et al. (2013) Endoglin (CD105) expression on microvessel endothelial cells in juvenile nasopharyngeal angiofibroma: tissue microarray analysis and association with prognostic significance. Head Neck 35: 1719-1725.

12. Zhang M, Sun X, Yu H, Hu L, Wang D (2011) Biological distinctions between juvenile nasopharyngeal angiofibroma and vascular malformation: An immunohistochemical study. Acta Histochem 113: 626-630.

13. Pauli J, Gundelach R, Vanelli-Rees A, Rees G, Campbell C, et al. (2008) Juvenile nasopharyngeal angiofibroma: an immunohistochemical characterisation of the stromal cell. Pathology 40: 396-400.

14. Ponti G, Losi L, Pellacani G, Rossi GB, Presutti L, et al. (2008) Wnt pathway, angiogenetic and hormonal markers in sporadic and familial adenomatous polyposis-associated juvenile nasopharyngeal angiofibromas (JNA). App Immunohistochem Mol Morphol 16: 173-178.

15. Sun $Y$, Wu Z (2006) Expression of PCNA, VEGF and bFGF in endothelial cells of juvenile nasopharyngeal angiofibroma. Lin Chuang Er Bi Yan Hou Ke Za Zhi 20: $1076-1078$

16. Brieger J, Wierzbicka M, Sokolov M, Roth Y, Szyfter W, et al. (2004) Vesse density, proliferation and immunolocalization of vascular endothelial growth factor in juvenile nasopharyngeal angiofibromas. Arch Otolaryngol Head Neck Surg 130: 727-731.

17. Gautham K, Ogale SB, Shraddha RU, Ajay D (2002) Expression of GSTM1 in angiofibromas. J Laryngol Otol 116: 352-354.

18. Brentani MM, Butugan O, Oshima CTF, Torloni H, Paiva LJ (1989) Multiple steroid receptors in nasopharyngeal angiofibromas. Laryngoscope 99: 398-401.

19. Lee DA, Rao BR, Meyer JS, Prioleau PG, Bauer WC (1980) Hormonal receptor determination in juvenile nasopharyngeal angiofibromas. Cancer 46: 547-551.

20. Hwang HC, Mills SE, Patterson K, Gown AM (1998) Expression of androgen receptors in nasopharyngeal angiofibroma: an immunohistochemical study of 24 cases. Mod Pathol 11: 1122-1126.

21. Farag MM, Ghanimah SE, Ragaie A, Saleem TH (1987) Hormonal receptors in juvenile nasopharyngeal angiofibroma. Laryngoscope 97: 208-211.

22. Gatalica Z (1998) Immunohistochemical analysis of steroid hormone receptors in nasopharyngeal angiofibromas. Cancer Lett 127: 89-93.

23. Liu Z, Wang J, Wang H, Wang D, Hu L, et al. (2015) Hormonal receptors and vascular endothelial growth factor in juvenile nasopharyngeal angiofibroma. Acta Otolaryngol 135: 51-57

24. Montag AG, Tretiakova M, Richardson M (2006) Steroid hormone receptor expression in nasopharyngeal angiofibromas. Consistent expression of estrogen receptor beta. Am J Clin Pathol 125: 832-837.

25. Schuon R, Brieger J, Heinrich UR, Roth Y, Szyfter W, et al. (2007) Immunohistochemical analysis of growth mechanisms in juvenile angiofibroma. Eur Arch Otorhinolaryngol 264: 389-394.

26. Schiff M, Gonzalez A, Ong M, Baird A (1992) Juvenile nasopharyngea angiofibroma contain an angiogenic growth factor: basic FGF. Laryngoscope 102: 940-945.

27. Nagai MA, Butugan O, Logullo A, Brentani MM (1996) Expression of growth factors, protooncogenes and p53 in nasopharyngeal angiofibromas. Laryngoscope 106: 190-195.

28. Schick B, Wemmert S, Jung V, Steuder W, Montenarh M, et al. (2006) Genetic heterogeneity of MYC oncogene in advanced juvenile angiofibromas. Cancer Genet Cytogenet 164: 25-31.

29. Dillard DG, Cohen C, Muller S, Del Gaudio J, Reichman O, et al. (2000) Immunolocalization of activated transforming growth factor beta1 in juvenile nasopharyngeal angiofibroma. Arch Otolaryngol Head Neck Surg 126: 723-735.

30. Rippel C, Plinkert PK, Schick B (2003) Expression of members of the cadherincatenin-protein family in juvenile angiofibromas. Laryngorhinootologie 82: 353-357.

31. Abraham SC, Montgomery EA, Giardiello FM, Wu TT (2001) Frequent betacatenin mutations in juvenile nasopharyngeal angiofibromas. Am J Pathol 158 1073-1078.

32. Schick B, Veldung B, Wemmert S, Jung V, Montenarh M, et al. (2005) p53 and Her-2/neu in juvenile angiofibromas. Oncol Rep 13: 453-457.

33. Maniglia MP, Ribeiro ME, Costa NM, Jacomini ML, Carvalho TB, et al. (2013) Molecular pathogenesis of juvenile nasopharyngeal angiofibroma in Brazilian patients. Pediatr Hematol Oncol 30: 616-622.

34. Schick B, Wemmert S, Bechtel U, Nicolai P, Hofmann T, et al. (2007) Comprehensive genomic analysis identifies MDM2 and AURKA as nove amplified genes in juvenile angiofibromas. Head Neck 29: 479-487.

35. Coutinho CM, Bassini AS, Gutiérrez LG, Butugan O, Kowalski LP, et al. (1999) Genetic alterations in Ki-ras and Ha-ras genes in juvenile nasopharyngeal angiofibromas and head and neck cancer. Sao Paulo Med J 117: 113-120.

36. Duerr S, Wendler O, Aigner T, Karosi S, Schick B (2008) Metalloproteinases in juvenile angiofibroma - A collagen rich tumor. Hum Pathol 39: 259-268.

37. Sun X, Guo L, Wang J, Wang H, Liu Z, et al. (2014) Prognostic value of matrix metalloproteinase 9 expression in patients with juvenile nasopharyngea angiofibroma: Tissue microarray analysis. Int J Pediatr Otorhinolaryngol 78 1232-1238

38. Sun X, Guo L, Wang H, Yu H, Wang J, et al. (2014) The presence of tumorinfiltrating IL-17-producing cells in juvenile nasopharyngeal angiofibroma tumor microenvironment is a poor prognostic factor. Am J Otolaryngol 35: 582-588.

39. Kumagami $H$ (1991) Testosterone and estradiol in juvenile nasopharyngea angiofibroma tissue. Acta Otolaryngol 111: 569-573.

40. Kumagami H (1993) Sex hormones in juvenile nasopharyngeal angiofibroma tissue. Auris Nasus Larynx 20: 131-135.

41. Valanzano R, Curia MC, Aceto G, Veschi S, De Lellis L, et al. (2005) Genetic evidence that juvenile nasopharyngeal angiofibroma is an integral FAP tumour Gut 54: 1046-1047.

42. Mishra A, Singh V, Verma V, Pandey S, Trivedi R, et al. (2016) Current status and clinical correlation of beta-catenin in juvenile nasopharyngeal Angiofibroma J Laryngol Otol 30: 1-7. 